\title{
Optical fiber Bragg grating- instrumented silicone liner for interface pressure measurement within prosthetic sockets of lower- limb amputees
}

Ebrahim Al-Fakih

Nooranida Arifin

Gholamhossein Pirouzi

Faisal Rafiq Mahamd Adikan

Hanie Nadia Shasmin

Noor Azuan Abu Osman 


\title{
Optical fiber Bragg grating-instrumented silicone liner for interface pressure measurement within prosthetic sockets of lower-limb amputees
}

\author{
Ebrahim Al-Fakih, ${ }^{\mathrm{a}, *}$ Nooranida Arifin, ${ }^{\mathrm{a}}$ Gholamhossein Pirouzi, ${ }^{\mathrm{a}}$ Faisal Rafiq Mahamd Adikan, ${ }^{\mathrm{b}}$ \\ Hanie Nadia Shasmin, ${ }^{a}$ and Noor Azuan Abu Osman ${ }^{a}$ \\ aUniversity of Malaya, Department of Biomedical Engineering, Faculty of Engineering, Kuala Lumpur, Malaysia \\ bUniversity of Malaya, Department of Electrical Engineering, Faculty of Engineering, Kuala Lumpur, Malaysia
}

\begin{abstract}
This paper presents a fiber Bragg grating (FBG)-instrumented prosthetic silicone liner that provides cushioning for the residual limb and can successfully measure interface pressures inside prosthetic sockets of lower-limb amputees in a simple and practical means of sensing. The liner is made of two silicone layers between which 12 FBG sensors were embedded at locations of clinical interest. The sensors were then calibrated using a custom calibration platform that mimics a real-life situation. Afterward, a custom gait simulating machine was built to test the liner performance during an amputee's simulated gait. To validate the findings, the results were compared to those obtained by the commonly used F-socket mats. As the statistical findings reveal, both pressure mapping methods measured the interface pressure in a consistent way, with no significant difference $(P$-values $\geq 0.05)$. This pressure mapping technique in the form of a prosthetic liner will allow prosthetics professionals to quickly and accurately create an overall picture of the interface pressure distribution inside sockets in research and clinical settings, thereby improving the socket fit and amputee's satisfaction. $\odot 2017$ Society of Photo-Optical Instrumentation Engineers (SPIE) [DOI: 10.1117/1.JBO.22.8.087001]
\end{abstract}

Keywords: fiber Bragg grating sensors; interface pressure; biomechanics; prosthetic sockets; transtibial amputees; silicone liner. Paper 170221R received Apr. 7, 2017; accepted for publication Jul. 27, 2017; published online Aug. 19, 2017.

\section{Introduction}

Piezoresistive transducers, such as the F-socket system, are the only commercialized and most commonly used sensing technique for interface pressure measurements within prosthetic sockets. Engsberg et al. and Houston et al. were the first to investigate the interface pressure within below-knee sockets using such a technique. ${ }^{1,2}$ They reported optimistic findings on the potential use of F-socket mats in clinical settings. The F-socket sensing mats showed flexibility, good sensitivity, and ease of use. ${ }^{3}$ They, however, exhibited nonlinearity, hysteresis, drift, and susceptibility to electromagnetic interferences (EMIs), casting doubt on the research findings. ${ }^{4}$ Furthermore, these mats may wrinkle and malfunction during experiments, thus affecting the measurement accuracy. ${ }^{5}$ Other capacitiveand strain gauge-based techniques have also been proposed, which also exhibited several limitations that hampered their use in clinical settings. ${ }^{6,7}$ Therefore, it is deemed necessary to develop a new measurement technique based on the fiber Bragg grating (FBG) sensors that offers practical and reliable interface pressure measurement within prosthetic sockets with minimal limitations.

Recently, researchers introduced the optical FBG sensors as a potential alternative to the commercial F-socket system for pressure measurement at the residual limb-socket interface of below-knee prosthetic sockets. ${ }^{8}$ The prominent advantages of this technology are linearity, high accuracy and sensitivity, small size, negligible drift, mutiplexability, and immunity to

*Address all correspondence to: Ebrahim Al-Fakih, E-mail: engr.fakih@yahoo com the EMIs. ${ }^{9-11}$ Al-Fakih et al. implemented the first investigation into the potential use of FBG sensors in prosthetic applications. ${ }^{12}$ A customized FBG sensor was fabricated, characterized, and tested in a below-knee socket, demonstrating very good sensitivity and acceptable hysteresis, i.e., satisfied the requirement of precise measurement of the pressure applied to the patella tendon bar region of below-knee prosthesis. The same researchers further studied the repeatability of FBG sensors and found that the pressure values remained quite consistent during 15 repeated gait cycles, implying that the FBG sensors yielded accurate and consistent signals with no drift. ${ }^{8}$ This can possibly make them superior to the previous techniques. In addition, expandable FBG sensing pads were fabricated to measure interface pressures at eight subregions on the residual limb of an amputee, and the results were compared with F-socket mats for validation purposes, revealing that FBGs could successfully measure these interface pressures. However, higher pressure values were recorded by the FBG sensors compared with the F-socket mats, which was attributed to the thickness of the FBG sensing pads ( $3 \mathrm{~mm}$ ) compared with the F-socket sensing mats $(0.2 \mathrm{~mm})$.

In this paper, we introduce an innovative, customized FBGinstrumented silicone liner that eliminates the previous limitations and provides a simple and practical means of sensing. It is designed to cushion the typical loads applied by the socket to the residual limb and functions as a real-time interface pressure sensing tool. This design is meant to be used repeatedly in clinical and research settings, which makes it superior to

1083-3668/2017/\$25.00 @ 2017 SPIE 
F-socket mats that are usually discarded after the first utilization due to drift and calibration issues.

\section{Instrumented Silicone Liner Fabrication and Calibration}

\subsection{Mold Fabrication}

A customized FBG-instrumented liner was fabricated using an inner solid mold and an outer shell so as to inject the silicone materials in between, thereby forming the intended prosthetic silicone liner. First, a gypsum cone-shaped positive cast was made to form the inner solid mold with a height of $42 \mathrm{~cm}$ and a radius of $5.25 \mathrm{~cm}$ at the proximal part, tapering to a radius of $3.75 \mathrm{~cm}$ at the distal end [Fig. 1(a)]. To maintain the required space between the inner mold and outer shell, a commercially available silicone liner was rolled over the inner mold, followed by an additional layer of stockinet [Fig. 1(a) right]. This assembly was then mounted onto a vacuum holding jig. Subsequently, two 5-mm-thick polypropylene sheets, each of which constructed half of the outer shell, were heated up in the oven to a temperature of $140^{\circ} \mathrm{C}$ for about $15 \mathrm{~min}$. Then, these two sheets were put together along the assembly to form a conical-shaped outer shell as shown in Fig. 1(b) left. The excess plastic was trimmed, and the vacuum was maintained during the whole process. The two shell halves were left in place until they completely cooled down. A few holes were drilled along the sides of the shells before disassembling it in order to put the two halves back together when fabricating the FBG-instrumented silicone liner [Fig. 1(b) right].

\subsection{Custom Fabrication of FBG-Instrumented Sensing Liner}

The FBG-instrumented liner was made of two layers of silicone materials (inner and outer) with 12 FBG elements embedded in between. First, silicone with shore hardness-A 20 was evenly brushed directly over the surface of the inner gypsum mold to form a 1-mm-thick silicone layer [Fig. 2(a)]. A total of 12 FBG elements were placed in a special configuration over the inner silicone layer in locations of clinical interest, such
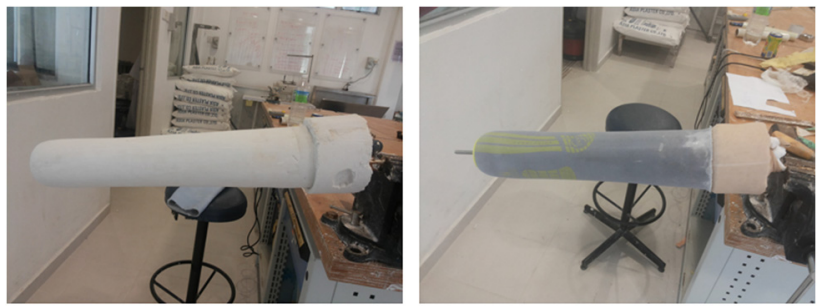

(a)
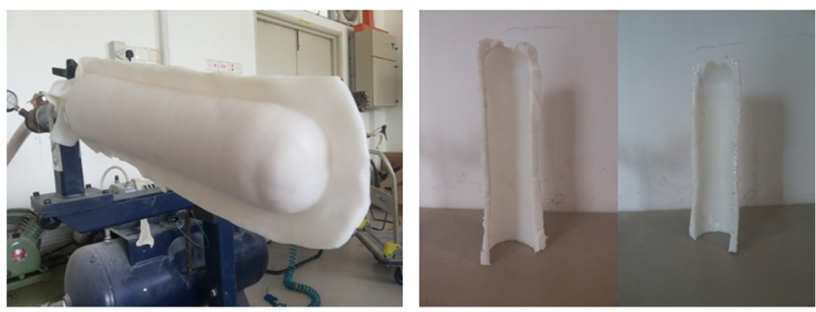

(b)

Fig. 1 Mold fabrication. (a) Inner mold fabrication and modification and (b) outer shell made up of two halves. as the proximal, middle, and distal regions of all four aspects (anterior, posterior, medial, and lateral) of the limb mold [Fig. 2(b)]. Beforehand, each of these FBGs was sandwiched between two thin layers of stockinet for utmost protection as shown in the inset of Fig. 2(b). Then, these sensing pads were transversally attached over the surface of the inner silicone layer using special silicone glue. Subsequently, the outer layer was fabricated using the injection molding technique. This was performed by assembling the two halves of the outer shell over the first inner layer of silicone and FBG sensing pads [Fig. 2(c)]. Guidance grooves were carved out to keep the outer shell in place and to maintain the required space between the inner

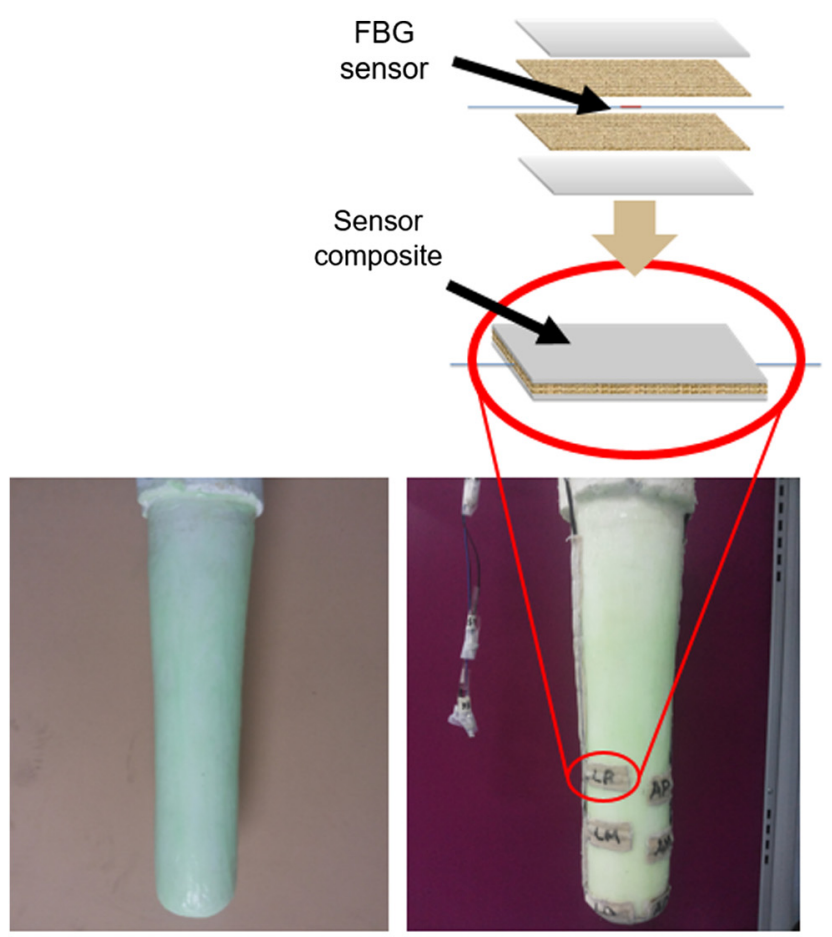

(a)
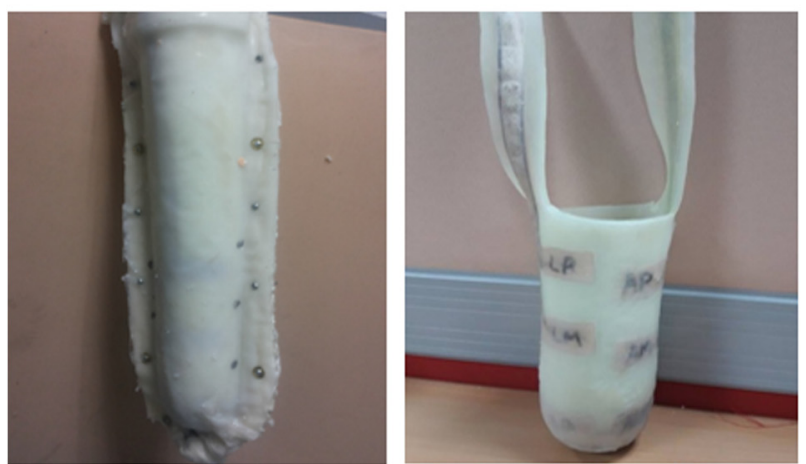

(b)

Fig. 2 Fabrication procedures for the sensing silicone liner. (a) Brushing a 1-mm-thick layer of silicone over the inner mold, (b) attaching the 12 sensors on the first silicone layer at locations of interest represented by the abbreviations: AP, anterior proximal; $\mathrm{AM}$, anterior middle; $\mathrm{AD}$, anterior distal; $\mathrm{LP}$, lateral proximal; $\mathrm{LM}$, lateral middle; LD, lateral distal; MP, medial proximal; MM, medial middle; MD, medial distal; PP, posterior proximal; PM, posterior middle; and PD, posterior distal, (c) assembling the two halves of the outer shell and then injecting the silicone material, and (d) an image showing the final FBG-instrumented liner after disassembling the outer shell and trimming the excess parts. 


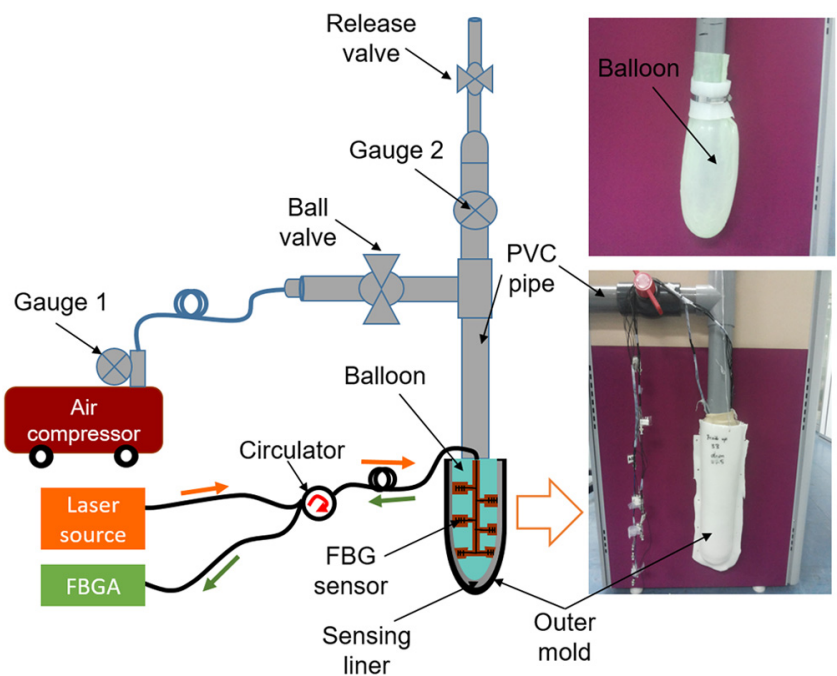

Fig. 3 Calibration platform for the FBG-instrumented liner. The two insets show real images of the fabricated balloon and the outer mold used for the calibration procedure.

silicone layer and outer shell. Silicone with shore hardness-A 5 was then injected into this space through a small hole drilled at the distal end of the shell to ensure that no bubbles would develop during the injection. After $24 \mathrm{~h}$, the outer shell was disassembled and the FBG-instrumented liner was ready to use [Fig. 2(d)].

\subsection{Sensing Liner Calibration}

A custom experimental setup was prepared for the sensing liner calibration as shown in Fig. 3. The sensing liner was calibrated in a way that mimics the actual environment of socket interface. A heavy-duty balloon with a 1-mm-thick wall was fabricated and then inserted into the sensing liner, which was, in turn, placed inside a conical-shaped hollow mold made especially for this setup as shown in Fig. 3. The balloon was then connected to an air compressor using a PVC pipe that contained a pressure gauge to indicate the pressure supplied to the balloon. The fiber pigtail of each of the 12 FBG sensing elements was coupled into a broadband light source, and the shift in the

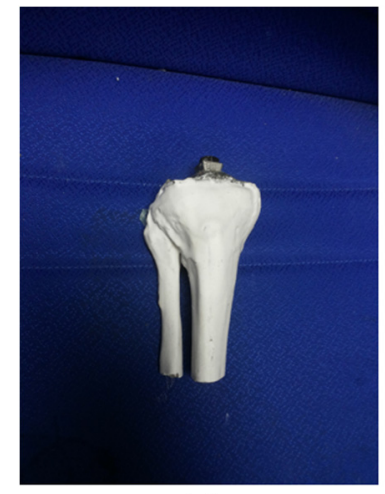

(a)

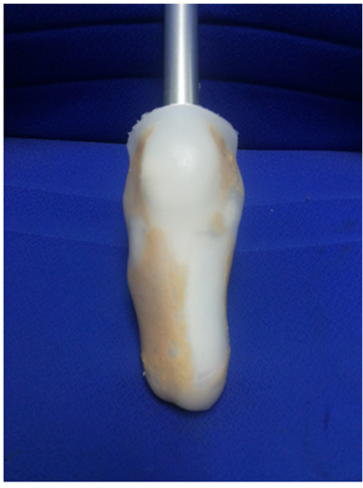

(b)
Fig. 4 Artificial residual limb fabrication. (a) A replica of the upper parts of the bones; tibia and fibula. (b) Final artificial residual limb. The brown silicone represents the muscle tissues while the white soft silicone represents the skin. reflected Bragg wavelengths was determined using a fiber Bragg grating analyzer (FBGA, Bayspec). The compressor applied ascending and descending air pressure into the balloon in five experimental trials with a dynamic range starting from 0 up to $30 \mathrm{kPa}$ and then gradually decreasing down to $0 \mathrm{kPa}$. The resultant shifts in the Bragg wavelength of the 12 FBGs were recorded accordingly.

\section{Below-Knee Prosthetic Device Development and Production}

\subsection{Artificial Residual Limb Fabrication}

No subject participated in this study; therefore, attempts were made to mimic a real-life situation by making a replica of an

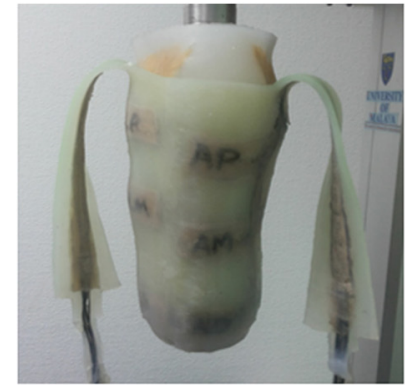

(a)

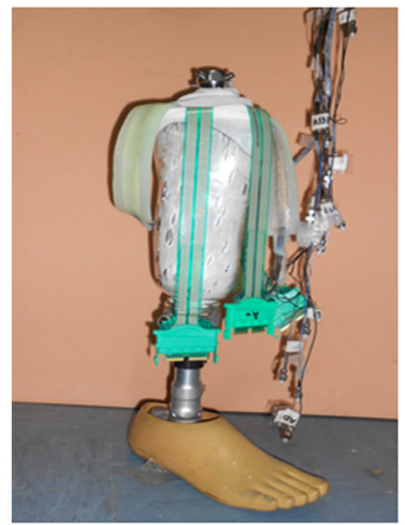

(c)

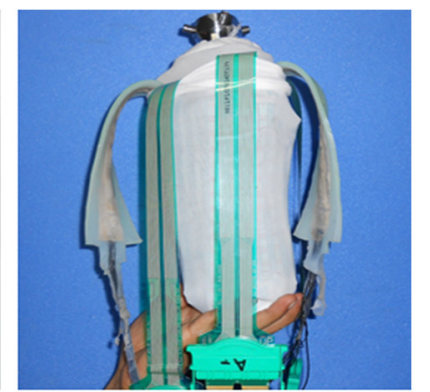

(b)

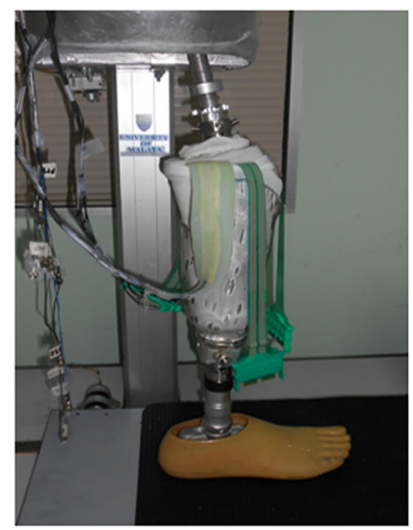

(d)

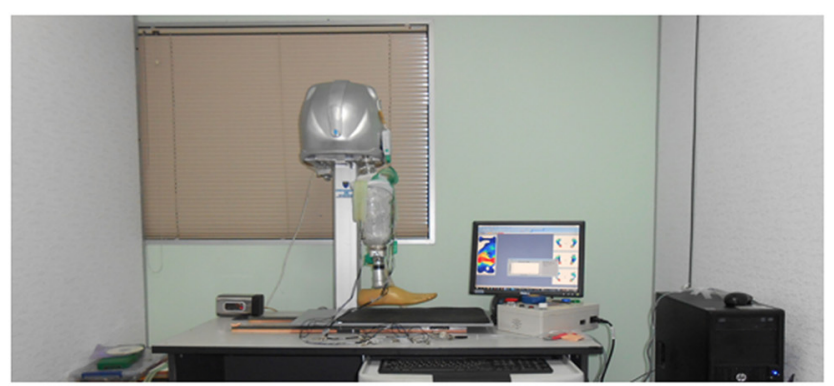

(e)

Fig. 5 The experimental setup for interface pressure measurements using both techniques, FBG sensors and F-socket mats. (a) The FBGinstrumented liner was rolled over the artificial residual limb. (b) Four F-socket mats were then adhered to the liner and covered up by a thin stockinet. (c) and (d) The installation of both sensing techniques in the prosthesis assembly. (e) The prosthesis assembly was suspended to the gait simulating machine, and the machine is running. 
amputee's residual limb. The actual residual limb contains bones (upper parts of tibia and fibula), muscles, and skin. Hence, a replica of the upper parts of the tibia and fibula bones was made from plastic polyester resin as shown in Fig. 4(a). The muscles and skin were made of silicone with 20 and 10 shore A hardness, respectively. Figure 4(b) shows the final limb model.
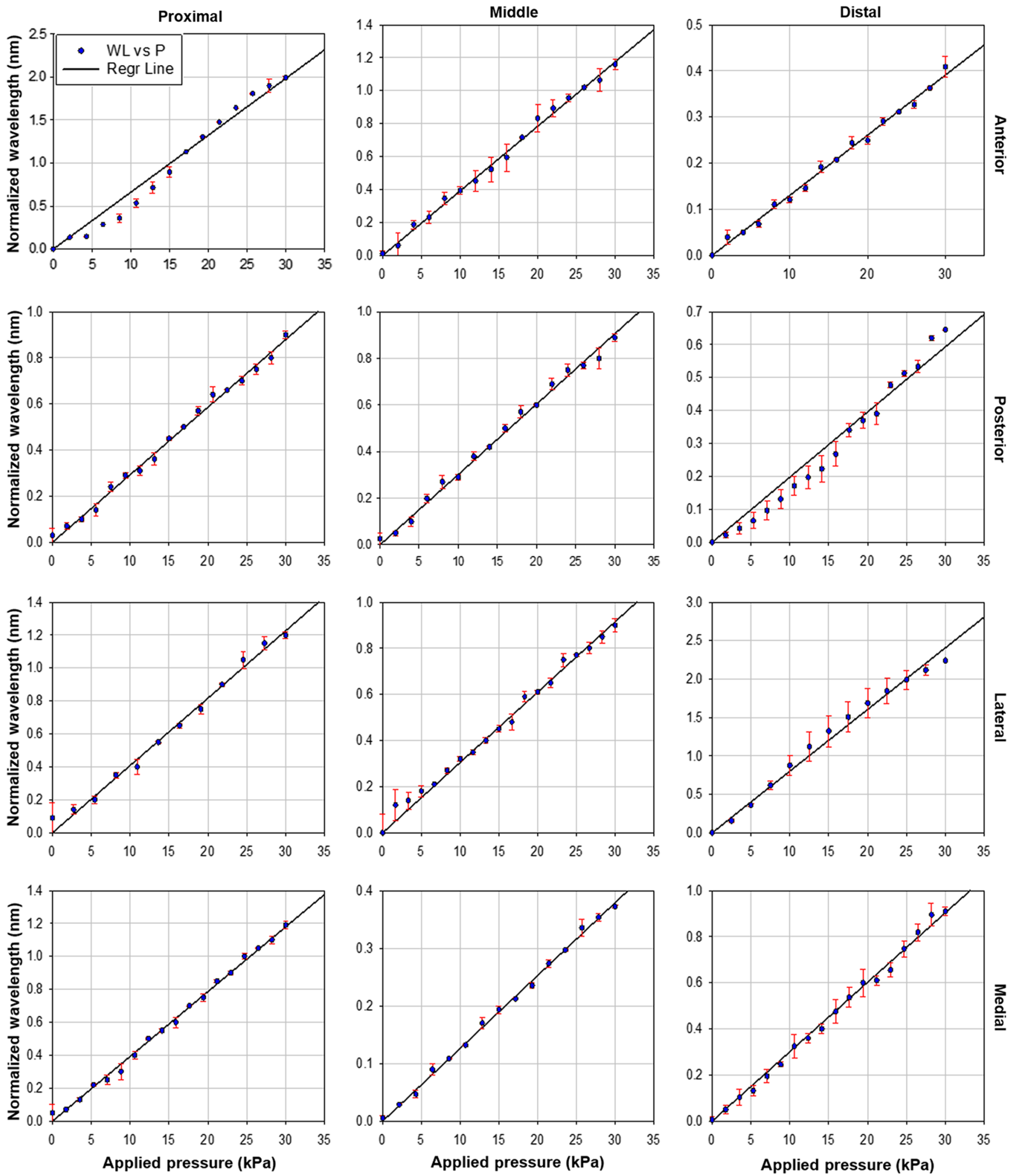

Fig. 6 The calibration curves of 12 FBG sensors showing their linear response. 
cast. Third, the positive mold was modified to acquire the final shape of the socket. Finally, a 12-mm-thick polycarbonate plastic sheet was heated up in the oven to a temperature of $150^{\circ} \mathrm{C}$ for about $20 \mathrm{~min}$ and then draped over the positive mold and left for a couple of minutes to cool down. The excess plastic was trimmed, so the socket conformed to the intended residual limb shape.

\section{Amputee Gait Simulating Machine}

To test our sensing liner, a machine (patent pending) was designed and built to mimic the amputee's gait cycle. The prosthetic device fabricated in the previous steps can be suspended by an aluminum pylon that sways back and forth by a motor mounted onto the machine head (Fig. 5). The prosthesis foot touches a sliding panel below, allowing a complete stance (heel strike, midstance, and toe-off) and swing phases of gait.

\section{Experimental Setup and Procedures}

To set up the prosthetic device on the gait simulating machine for the experiments, the FBG-instrumented liner was rolled over the artificial residual limb [Fig. 5(a)]. For comparison purposes, the commercially available F-socket mats were also employed for pressure measurements at the same sensing regions to simultaneously compare the results obtained using the two sensing techniques [Fig. 5(b)]. The F-socket mats were trimmed according to the contours of the residual limb and placed on the anterior, posterior, medial, and lateral aspects of the residual limb. Adhesive spray was used to keep the F-socket mats firmly onto the artificial residual limb to avoid displacement. Prior to this setup and experiments, the F-socket mats and FBG sensors were calibrated. The prosthetic device was then firmly suspended to the gait simulating machine. Figure 5(c) shows the installation of the FBG-instrumented liner and F-socket mats in the prosthesis.
The pressure profile was determined as a function of the recorded wavelength shifts. Therefore, the pigtail of each FBG sensor was coupled into a broadband light source and the wavelength shifts that occurred due to the pressure applied during the gait cycle were recorded by the FBGA. A LabVIEW program capable of logging only the coordinates of the peak wavelengths of the reflected spectra at a sampling frequency of $50 \mathrm{~Hz}$ for each FBG was developed. Similarly, four F-socket mats were installed over the FBG-instrumented liner covering all the socket aspects, and their ends were connected to the Tekscan system [Fig. 5(d)].

Once the gait simulating machine was turned on, the FBGA device determined the backreflected wavelengths in real-time. Simultaneously, the Tekscan system recorded the pressure applied to the socket by the residual limb replica to create an overall picture of the interface pressure distribution in the below-knee socket.

\section{Results}

\subsection{Liner Characterization}

Following the preparation of the custom experimental setup for the calibration, the 12 FBG sensors were coupled into a light source and the shifts in the backreflected Bragg wavelength were recorded using the FBGA device. Since each FBG sensor was subjected to ascending and descending air pressure in five experimental trials, the obtained five calibration curves were averaged and the standard deviations were calculated to interpret the sensor behavior. Figure 6 shows the calibration curves and standard deviations of all 12 FBG sensing elements with regression lines. From the regression lines, the calibration equations can be simply obtained and the sensors' sensitivity can be calculated. Table 1 summarizes the sensors' sensitivities, calibration equations, and calibration dynamic ranges. It is obvious that all FBG sensors have shown a linear relationship between the

Table 1 Twelve FBG sensors' sensitivities, dynamic ranges, and calibration equations.

\begin{tabular}{|c|c|c|c|c|c|c|}
\hline \multirow[b]{2}{*}{ Region } & \multirow[b]{2}{*}{ Sensor site } & \multirow[b]{2}{*}{ Sensitivity (kPa/nm) } & \multicolumn{3}{|c|}{ Calibration dynamic range } & \multirow[b]{2}{*}{ Equations } \\
\hline & & & Min. WL (nm) & Max. WL (nm) & Max. WL shift $(\mathrm{nm})$ & \\
\hline \multirow[t]{3}{*}{ Anterior } & Proximal & 15.12 & 1550.23 & 1552.22 & 1.99 & $P=15.12 \mathrm{WL}$ \\
\hline & Middle & 25.51 & 1550.19 & 1551.35 & 1.16 & $P=25.51 \mathrm{WL}$ \\
\hline & Distal & 76.69 & 1539.95 & 1540.36 & 0.41 & $P=76.69 \mathrm{WL}$ \\
\hline \multirow[t]{3}{*}{ Posterior } & Proximal & 34.12 & 1550.31 & 1551.21 & 0.90 & $P=34.12 \mathrm{WL}$ \\
\hline & Middle & 30.11 & 1554.92 & 1555.81 & 0.89 & $P=30.11 \mathrm{WL}$ \\
\hline & Distal & 50.53 & 1556.12 & 1556.77 & 0.65 & $P=50.53 \mathrm{WL}$ \\
\hline \multirow[t]{3}{*}{ Lateral } & Proximal & 24.45 & 1549.72 & 1550.92 & 1.20 & $P=24.45 \mathrm{WL}$ \\
\hline & Middle & 32.76 & 1547.23 & 1548.13 & 0.90 & $P=32.76 \mathrm{WL}$ \\
\hline & Distal & 12.46 & 1549.55 & 1551.79 & 2.24 & $P=12.46 \mathrm{WL}$ \\
\hline \multirow[t]{3}{*}{ Medial } & Proximal & 25.41 & 1555.79 & 1556.98 & 1.19 & $P=25.41 \mathrm{WL}$ \\
\hline & Middle & 79.10 & 1555.11 & 1555.48 & 0.37 & $P=79.10 \mathrm{WL}$ \\
\hline & Distal & 33.18 & 1550.09 & 1551.00 & 0.91 & $P=33.18 \mathrm{WL}$ \\
\hline
\end{tabular}


Table 2 Peak pressure values $(\mathrm{kPa})$ at the residual limbs regions during the stance phase.

\begin{tabular}{|c|c|c|c|c|}
\hline \multirow[b]{2}{*}{ Region } & \multirow[b]{2}{*}{ Subregion } & \multicolumn{2}{|c|}{ Mean peak pressure (SD) } & \multirow[b]{2}{*}{$P$-value } \\
\hline & & F-socket sensor & FBG sensors & \\
\hline \multirow[t]{3}{*}{ Anterior } & Proximal & $17.23(1.23)$ & $16.52(0.99)$ & 0.05 \\
\hline & Middle & $34.18(4.80)$ & $35.56(4.69)$ & 0.36 \\
\hline & Distal & $-^{a}$ & $17.06(1.16)$ & $-^{a}$ \\
\hline \multirow[t]{3}{*}{ Posterior } & Proximal & $42.25(1.75)$ & $43.03(1.99)$ & 0.22 \\
\hline & Middle & $31.07(1.78)$ & 30.95 (1.62) & 0.84 \\
\hline & Distal & $18.90(1.47)$ & $19.72(1.13)$ & 0.86 \\
\hline \multirow[t]{3}{*}{ Lateral } & Proximal & $23.92(1.03)$ & $23.47(0.97)$ & 0.17 \\
\hline & Middle & $35.26(0.97)$ & $34.94(0.94)$ & 0.34 \\
\hline & Distal & $53.99(1.32)$ & $54.22(1.03)$ & 0.55 \\
\hline \multirow[t]{3}{*}{ Medial } & Proximal & $38.38(2.19)$ & $38.29(2.05)$ & 0.8 \\
\hline & Middle & $12.67(1.47)$ & $12.69(0.87)$ & 0.97 \\
\hline & Distal & $56.06(2.44)$ & $56.16(2.54)$ & 0.89 \\
\hline
\end{tabular}

${ }^{a}$ The FBG sensor at the anterior distal region failed during the experiments, thus no data at that subregion was recorded and $P$-value cannot be calculated.

shifts in the backreflected wavelengths and the applied pressures, which is in agreement with the mechanical and optical properties of FBGs. ${ }^{8}$

\subsection{Interface Pressure Profile Using the Two Measurement Techniques}

The interface peak pressures measured with the newly developed FBG sensing liner were compared with the results of pressure profile during gait simulation by the commonly used Fsocket sensing mats. Statistical analysis in the form of a $t$-test was applied to the data obtained from 10 cycles of gait simulation by the gait simulating machine. The mean peak pressures for various regions of the residual limb were compared, and $P$ values were more than 0.05 (Table 2), meaning that both mapping methods measured the pressure in a consistent way and with no significant difference. The bar chart in Fig. 7 shows the mean peak pressure values at the four major aspects of the residual limb during the stance phase of gait. The peak pressure patterns recorded by the FBG sensing liner and F-socket mats over all 12 subregions of the residual limb during a complete gait cycle (stance and swing phases) are shown in Fig. 8. The results show that the new sensing liner is reliable for measuring pressures within the prosthesis socket.

\section{Discussion}

Several researchers have assessed the validity and reliability of F-socket transducers for prosthetic use in a clinical environment. ${ }^{13-15}$ Hachisuka et al. studied the performance of Tekscan pressure transducers when subjected to rapid and repetitive movements and reported sensor drift, hysteresis, and temperature sensitivity. ${ }^{14}$ For accuracy purposes, it is recommended that the transducers be equilibrated a few days before the experiments. ${ }^{16}$ This process reduced the amount of drift that may occur in the transducers before they were used. Hysteresis, repeatability, and accuracy are therefore key issues. On the other hand, FBG sensors have shown excellent linearity, repeatability, accuracy, negligible drift, and acceptable hysteresis error. ${ }^{5}$ As the statistical findings reveal, the FBG can offer the same sensitivity and accuracy as the F-socket sensors, while solving the problems of drift and nonlinearity.

As Fig. 8 shows, during the stance phase of gait, the FBG sensing liner exhibits more linear results than the F-socket, which is obvious from the smooth curves. Since F-socket mats are resistive, their response is nonlinear, even though over a small range it seems almost linear. ${ }^{17}$ Moreover, what makes FBG sensors superior to the F-socket is that the newly developed FBG-instrumented liner could be used repeatedly during experiments on amputee subjects, without significant effect on the measurement accuracy. From our experience, the F-socket mats are only used once per experiment per subject as they crease and some sensors may fail, making them unusable for repeated applications. As the FBG technique was developed and designed in the form of a prosthetic liner, the amputee can easily and quickly wear it during the experiment.

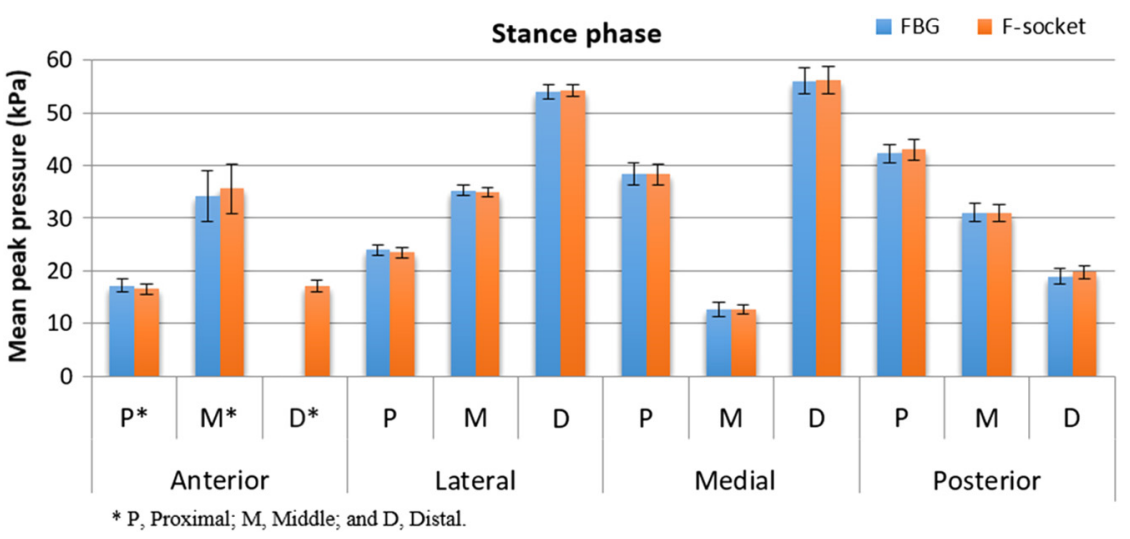

Fig. 7 Mean peak pressures at the major aspects of the residual limb recorded during the stance phase of gait. 

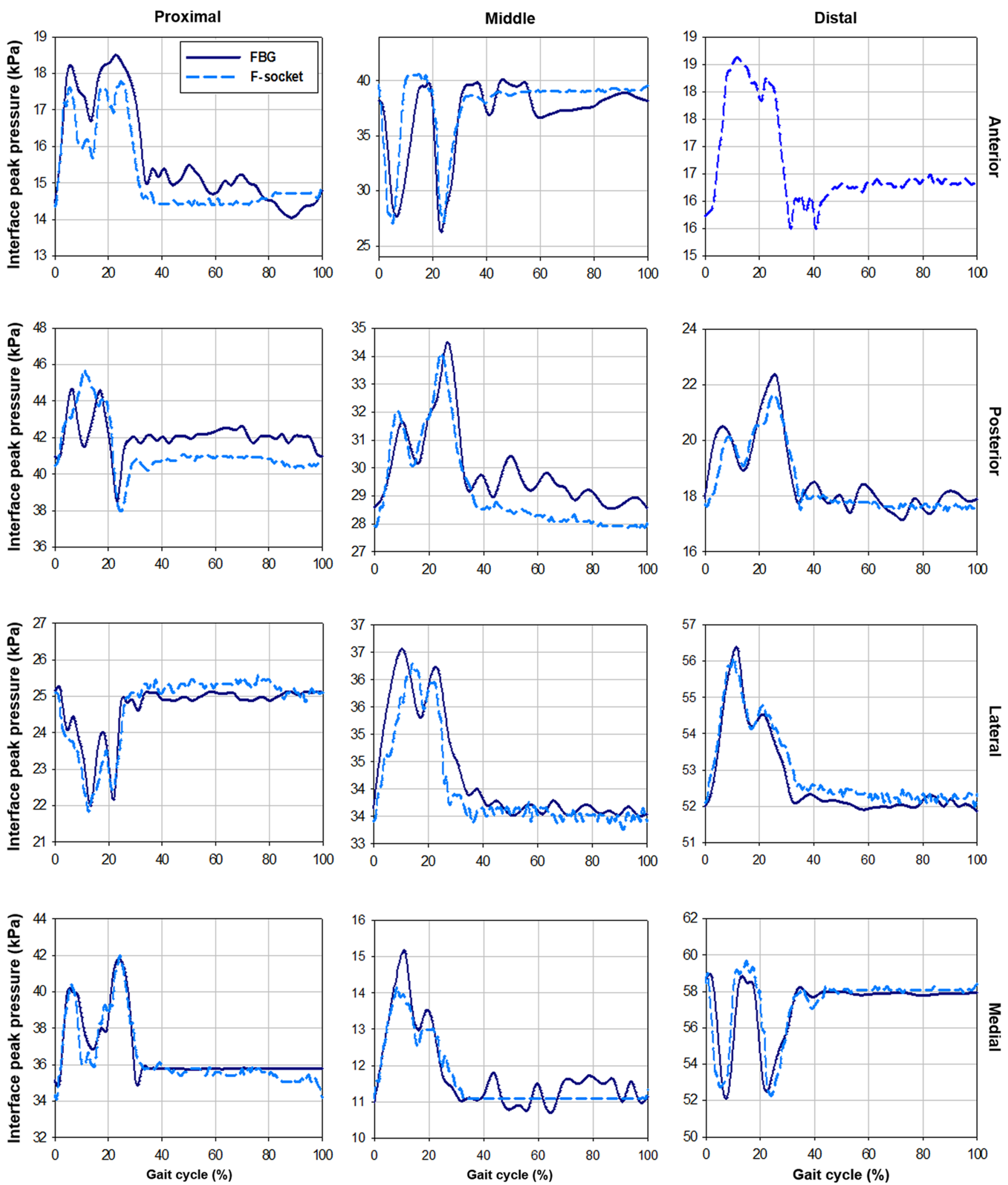

Fig. 8 The interface peak pressures at 12 subregions of the residual limb during a complete gait cycle using FBG sensing liner and F-socket transducers.

Another advantage of the FBG-instrumented liner as compared to the F-socket mats is that it is possible to monitor and measure pressures all over the residual limb, while each F-socket mat has a certain size of $20.3 \mathrm{~cm} \times 7.6 \mathrm{~cm}$. Thereby, it might not be enough to cover the whole residual limb for amputees with a larger circumference of the residual limb. Researchers, therefore, use four F-socket mats simultaneously, which affects the cost. However, FBG-instrumented liners, once mass produced in different sizes, can cover the whole residual limb and conform well to its surface, which sometimes has an 
irregular shape. Also, in the future, it will be possible to enhance the hardware system of FBG sensors in a way to monitor socket pressure during amputee's daily activities, not only in the laboratory.

\section{Conclusions}

The study findings suggest that the FBG-instrumented silicone liner can be used successfully as an alternative measurement means for interface pressure within the prosthetic socket. The main advantages are the lower cost of application, higher flexibility and conformity to the residual limb, less or no drift, and negligible hysteresis; additionally, repeated loading is possible without affecting the data. The comparison to the popular F-socket mats reveals good reliability and repeatability of pressure measurements. These findings indicate that FBG sensors are worth being explored more in clinical settings to further validate their merits.

\section{Disclosures}

The authors have no potential conflicts of interest to disclose.

\section{Acknowledgments}

This work was supported by the University of Malaya Research Fund Assistance (Grant No. BK101-2016).

\section{References}

1. J. Engsberg, J. Springer, and J. Harder, "Quantifying interface pressures in below-knee-amputee sockets," J. Assoc. Child. Prosth-Orthot Clin. 27, 81-88 (1992).

2. V. Houston et al., "Preliminary results with the DVA-Tekscan BK prosthetic socket/residual limb stress measurement system," in Proc. of 20th Annual Meeting of American Academy of Orthotist and Prosthetist, pp. 8-9 (1994).

3. A. M. Almassri et al., "Pressure sensor: state of the art, design, and application for robotic hand," J. Sens. 2015(1), 846487 (2015).

4. G. Pirouzi et al., "Review of the socket design and interface pressure measurement for transtibial prosthesis," Sci. World J. 2014(1), 849073 (2014).

5. E. A. Al-Fakih, N. A. Abu Osman, and F. R. Mahmad Adikan, "Techniques for interface stress measurements within prosthetic sockets of transtibial amputees: a review of the past 50 years of research," Sensors 16(7), 1119 (2016).

6. P. Saccomandi et al., "Microfabricated tactile sensors for biomedical applications: a review," Biosensors 4(4), 422-448 (2014).

7. J. Sanders, "Interface mechanics in external prosthetics: review of interface stress measurement techniques," Med. Biol. Eng. Comput. 33(4), 509-516 (1995).

8. E. Al-Fakih et al., "Development and validation of fiber Bragg grating sensing pad for interface pressure measurements within prosthetic sockets," IEEE Sens. J. 16(4), 965-974 (2016).

9. M. F. Domingues et al., "Insole optical fiber Bragg grating sensors network for dynamic vertical force monitoring," J. Biomed. Opt. 22(9), 091507 (2017).

10. L. Ding, X. Tong, and L. Yu, "Quantitative method for gait pattern detection based on fiber Bragg grating sensors," J. Biomed. Opt. 22(3), 037005 (2017).

11. E. Al-Fakih, N. A. Abu Osman, and F. R. Mahamd Adikan, "The use of fiber Bragg grating sensors in biomechanics and rehabilitation applications: the state-of-the-art and ongoing research topics," Sensors 12(10), 12890-12926 (2012).

12. E. A. Al-Fakih et al., "The capability of fiber Bragg grating sensors to measure amputees' trans-tibial stump/socket interface pressures," Sensors 13(8), 10348-10357 (2013).
13. A. Buis and P. Convery, "Calibration problems encountered while monitoring stump/socket interface pressures with force sensing resistors: techniques adopted to minimise inaccuracies," Prosthet. Orthot. Int. 21(3), 179-182 (1997).

14. K. Hachisuka et al., "Properties of the flexible pressure sensor under laboratory conditions simulating the internal environment of the total surface bearing socket," Prosthet. Orthot. Int. 22(3), 186-192 (1998).

15. A. Polliack et al., "Laboratory and clinical tests of a prototype pressure sensor for clinical assessment of prosthetic socket fit," Prosthet. Orthot. Int. 26(1), 23-34 (2002).

16. W. Kim, D. Lim, and K. Hong, "An evaluation of the effectiveness of the patellar tendon bar in the trans-tibial patellar-tendon-bearing prosthesis socket," Prosthet. Orthot. Int. 27(1), 23-35 (2003).

17. J. M. Brimacombe et al., "Effect of calibration method on Tekscan sensor accuracy," J. Biomech. Eng. 131(3), 034503 (2009).

Ebrahim Al-Fakih has been a researcher at the Center for Applied Biomechanics, University of Malaya, Malaysia, since 2011. He received his $B S c$ degree in biomedical engineering from Hashemite University, Jordan, in 2008, and the MEng degree and PhD in biomedical engineering from the University of Malaya, Malaysia, in 2011 and 2017, respectively. His current research interests cover biomechanics, rehabilitation engineering, artificial limbs, and bioinstrumentation. He focuses particularly on the development of transtibial prosthetic sockets and liners.

Nooranida Arifin is a senior lecturer at the University of Malaya. She received her $\mathrm{BSc}$ degree and $\mathrm{PhD}$ in biomedical engineering from the University of Malaya and her MSc degree in prosthetics and orthotics from Eastern Michigan University, Michigan, USA. Her current research interests cover prosthetics and orthotics biomechanics, in particular, focusing on the balance mechanism with prosthetics intervention following lower-limb amputation. She is an active member of the International Society for Prosthetics Orthotics, Malaysia Chapter.

Gholamhossein Pirouzi received his BSc degree in industrial design from the University of Science and Technology, Iran, in 1999, his MBA degree in industrial management from the University of Tabriz, Iran, in 2011, and his MSc degree in biomechanics from the University of Malaya, Malaysia, in 2015; he is currently doing his PhD in biomechanics at the University of Malaya. His current research interests cover biomechanics, biomechatronics, and rehabilitation engineering, particularly focusing on developing a prosthetic gait simulator.

Faisal Rafiq Mahamd Adikan received his PhD from the Optoelectronics Research Centre, University of Southampton, United Kingdom, in 2007. His PhD work on flat fiber produced an international patent. Currently, he is the head of the Integrated Lightwave Research Group with the University of Malaya and is involved in developing innovative fabrication processes to incorporate optically active materials into a glass matrix. He has authored over 80 journal and conference papers in optics and engineering education.

Hanie Nadia Shasmin received her BEng degree in biomedical engineering from the University of Malaya, Malaysia, in 2006. She has been a research officer at the University of Malaya since 2012, where she is currently affiliated with the Centre for Applied Biomechanics, Faculty of Engineering.

Noor Azuan Abu Osman received his BEng degree in mechanical engineering from the University of Bradford, United Kingdom, and his MSc degree and PhD in bioengineering from the University of Strathclyde, United Kingdom. Currently, he is the dean of engineering and the deputy director of the Centre for Applied Biomechanics. He has authored books, conference proceedings, and journal articles. His main research interests are the measurements of human movement, prosthetics design, the development of instrumentation for forces and joint motion, and the design of prosthetics, orthotics, and orthopedic implants. 